\title{
Android Remote Monitoring System Based on loserver Data Collection
}

\author{
Linliang Zhang ${ }^{1, a^{*}}$, Peng Cheng ${ }^{2, b},{ }^{2, b}$ and ZhaoXia $\mathrm{Li}^{2, \mathrm{~b}}$ \\ ${ }^{1}$ Shanxi Transportation Research Institute, XueFu Street $79^{\text {th }}$, Taiyuan City, Shanxi Province, \\ China \\ ahanglinliang@163.com
}

Keywords: Android; Mobile Client; loserver; Monitoring

\begin{abstract}
In order to solve the remote Multi-Networks acquisition and real-time mobile monitoring of highway tunnel monitoring data, a kind of remote monitoring and data collection software design based on SCADA and Android was put forward. The system interact data with mobile intelligent terminal through the SCADA monitoring software for remote data acquisition. Through interconnect and interworking of multiple systems to realize mobile monitoring in real time, extend the compatibility of the system at the same time. By a variety of interface mode such as OPC, ODBC and SCADA API to realize interconnection with other systems or software, achieve multiple data collection. Meanwhile ensure the software compatibility and real-time performance through a variety of network connections and asynchronous transfer. Test results show that the proposed scheme successfully design the traditional tunnel monitoring and mobile real-time monitoring, has extensive application value.
\end{abstract}

\section{Introduction}

Tunnel electromechanical monitoring system is mainly used to monitor and control all the tunnels in the monitoring section. The real-time tunnel traffic conditions, the status of mechanical and electrical equipment and tunnel environment parameters and other information are monitored by it. The aim of tunnel electromechanical monitoring system is to solve the problem of control network mode and improve the intelligent level of remote control.

The implementation of tunnel electromechanical monitoring system has a variety of ways. The software system is generally configuration software. SCADA[1] platform is a way to achieve configuration software development. The tunnel monitoring system developed based on the SCADA platform is mainly used in the tunnel monitoring center, through which the mechanical and electrical facilities, traffic flow and events in the tunnel are monitored.

The remote monitoring of the tunnel can only be done in the monitoring room by Tunnel Monitoring Center. SCADA tunnel monitoring system cannot remotely monitor the running conditions of various equipments and tunnel incidents at a moving location[2]. When the emergency occurs in the tunnel, each staff member cannot get the early warning and prompts at the first time, and cannot remotely check the specific conditions in the tunnel either that cannot conduct long-range coordinated command. Therefore, monitoring through the tunnel monitoring room has many drawbacks, while the mobile terminal can monitor various facilities and emergencies in the tunnel at any time to facilitate viewing and remote operation. Thus, it has great importance for coordinated command and control management.

SCADA system is also called data acquisition and monitoring control system. It occupies an important position in the motion system. It can monitor and control field devices and realize many functions such as equipment control, communication, parameter adjustment, various signal alarms and data acquisition[3][4].

Android is the software platform and operating system of mobile terminal. The system can send data request and transmit data to remote data service, data acquisition system and data center from the terminal to realize remote on-line control and monitoring of equipment, parameters, alarms and events, etc.

In this paper, a remote monitoring and data acquisition system based on SCADA and Android is proposed. The system not only can realize the local control, remote monitoring and control of data, but 
also can carry on the data communication and control through the mobile terminal and SCADA system. The mobile terminal sends commands to control SCADA system. SCADA system receives the control commands and controls the corresponding electromechanical devices according to the control commands. The mobile terminal can also directly monitor and control the electromechanical devices by using the remote WebService service or the Sokect service, so as to realize the dual mode remote monitoring and control; mobile terminal has the functions, such as on-site troubleshooting, emergency handling and others.

\section{Overall framework design of the system}

Tunnel electromechanical monitoring system is an important department of tunnel monitoring center and information command center, and is directly related to the normal operation of the tunnel. The tunnel electromechanical monitoring system is responsible for monitoring all the mechanical and electrical equipment in the tunnel. When there is an emergency, gang control and automatic control can be done for the equipment according to the set threshold. The entire system data acquisition, equipment control, data transmission and data communication with the mobile terminal are completed according to the tunnel electromechanical equipment layout, data acquisition process, data control process, event feedback and so on. The system consists of various types of equipment controller, a variety of data communications, data acquisition interface, data storage, control terminals, Web services or Socket services, mobile terminals and other major components. The overall system data transfer process and communication is shown as follows:

Firstly, the main equipment communication method in the tunnel mainly includes the communication between equipment and PLC controller, the communication between the equipment and the upper computer through converting into digital signal with optical transceiver (mainly including serial communication and network port communication), as well as the communication between the main PLC and sub PLC[7].

Secondly, the upper computer data acquisition system is communicated with PLC controller through the driver and to communicate, and writes the driver according to the transmission protocol. The data acquisition system acquires data through the driver.

Thirdly, the data collected by the data acquisition system is transmitted to the monitoring platform for information exchange with the monitoring platform. The monitoring platform provides services for the smart App through the WebService. The smart App can request data and request remote control through the WebService interface.

\section{Data acquisition and data transmission system}

Tunnel mechanical and electrical monitoring system performs real-time acquisition of data and equipment monitoring and control through SCADA, and it can use a variety of networks (including optical communications, analog signal data communications, digital signal data communications, network, wireless, etc.) for data transmission. It can monitor tens of thousands of monitoring points, and can also interact with other software systems and hardware systems in various ways, for example, through ODBC, OPC, API, SEIVICE and so on[6].

The main method of data acquisition is to acquire data through IO driver in IOServer data acquisition unit. IO driver includes serial port driver, network port driver (including TCP and UDP driver) ; IOServer can load a variety of drivers to achieve multi-threaded multi-network data communications, to acquire data by serial port or network port driver.

SCADA system configures IOServer data acquisition unit through local path or remote path. SCADA system can also configure multiple IOServer data acquisition units and data backup, SCADA system can save the data to local historical database and implementation database, and can also save the data into an external database through ODBC, ADO or SCADA API and other ways; IOServer acquires data by the driver, while the acquired data is sent to the SCADA data variables with the form of timestamp. SCADA is displayed on the interface according to changes in data. 
In the runtime of IOServer data acquisition, there are at least two threads associated with the IOServer driver: the main thread and acquisition thread (which may have more than one). The acquisition thread scans all the variables in this thread at regular intervals. For the variable whose acquisition time is up, pack according to the packing rules processed in the driver to form a packet queue and processes the packet queues one by one until the packet queues is empty, and then determine whether the scanning cycle is up. If yes, scan immediately[5]. The data treatment process of IOServer data driver is shown as follows:

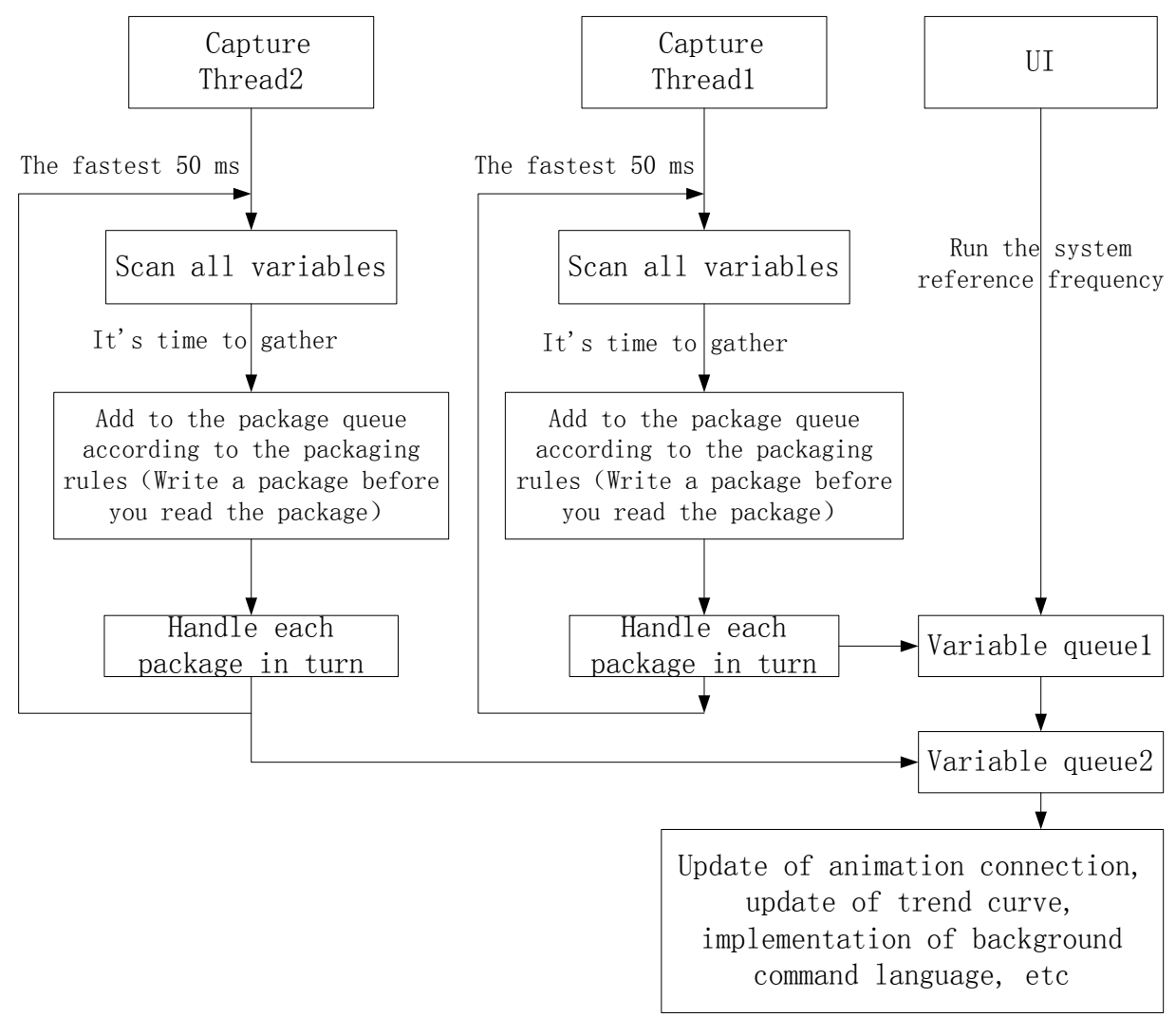

Figure 1. he data treatment process of IOServer data driver

The data acquisition speed in IOServer data acquisition process is related with three factors, namely code processing time, link transmission time, device response time, a packet processing time:

$\mathrm{T}_{\text {包 }}=$ Code processing time + link transmission time + device response time

So if there is more than one variable in a package, the acquisition time for each variable will be shortened. Now the condition that only one variable in a package is considered, the acquisition time of a variable is

$$
\mathrm{T}_{\text {var }}=\mathrm{T}_{\text {包 }}
$$

The actual acquisition cycle (Cvar) for a single variable depends not only on the acquisition frequency (set in the Kingview) but also on the sum of the processing times for all variables in the current thread package queue, namely:

Cvar $=\operatorname{Max}(50 *$ (acquisition frequency var/50) $+(($ acquisition frequency var\%50) $>0 ? 50: 0) \quad)$

From this, it can be seen that if the acquisition frequency of each variable in the project is not consistent, it is possible that the packet column length of the same variable is different every time, that is, the total processing time of all the variables in the current thread packet queue will be different, thus each actual refresh time for the variable will be affected.

From the above formula, we can see:

If the sum of the processing time for all variables in the current thread package queue < $50 *($ acquisition frequency var/50) $+(($ acquisition frequency var\%50) $>0$ ?50:0 $)$ the actual acquisition 
cycle of a single variable Cvar $=50 *($ acquisition frequency var/50) + ((acquisition frequency var\%50)>0?50:0)

If the sum of the processing time for all variables in the current thread package queue $>$ $50 *($ acquisition frequency var/50) + ((acquisition frequency var\%50)>0?50:0)

The actual acquisition cycle of a single variable Cvar $=$ the sum of the processing time of all the variables in the current thread package queue

From the above analysis, it can be seen that if a package contains multiple variables, the acquisition time of the variable can be greatly shortened, and the actual acquisition frequency of a single variable can be equal to the acquisition frequency set by the user.

The processing principle in the drive is: the one can be packaged should be packaged as much as possible. But the concrete realization must be based on the agreement.

Conventional packing rules are: the variables defined on the same device, the same register with the same data type are best to read the packaging. The maximum length of the packaging is generally 64 or 256 range. Writing generally does not need package. If the user writes a relatively large amount, and the speed is not required to be higher, we can give him a customized writing package. (Temperature control curve, etc.)

$\mathrm{Q}_{1}$. For the current package queue, a package cannot be read, after being failed to try to connect, how to deal with other packages?

If all packages for this device are not processed, just try according to the trying connection cycle; other packages are handled properly.

$\mathrm{Q}_{2}$. 8K variable structure, after overflow, covering the latest or the oldest?

First in first out. If overflow, cover the oldest.

$\mathrm{Q}_{3}$. The first package, does the acquisition frequency need to be seen?

Do not look at the acquisition frequency. Acquire and package according to ID number.

\section{Conclusion}

The designed data acquisition system can achieve the requirements of fast data acquisition and support serial communications and network communications. It can simultaneously communicate with a variety of devices and data transmission. The transmission has features of low latency and high speed. Multiple communication links can be set. Each link monitors the same equipment. Different links can meet the requirement of monitoring different equipments.

\section{Acknowledgements}

《" 'Internet + Highway Engineering "Construction Safety Management and Inspection Evaluation Te chnnology Research》, Project No.: 2017-1-24, Research Project of Transport Department of Shanx i Province .

\section{References}

[1] Accurate modeling of Modbus/TCP for intrusion detection in SCADA systems[J] . Niv Goldenberg,Avishai Wool. International Journal of Critical Infrastructure Protection . 2013 (2)

[2] Using Model-based Intrusion Detection for SCADA Networks. CHENG S, DUTERTRE B,FONG M,et al. SCADA Security Scientific Symposium . 2007

[3] "Networked Remote Meter Reading System based on Wireless Communication Technology". L.Cao,J.Tian,D.Zhang. proc.of IEEE International Conference on Information Acquisition . 2006

[4] Research on a Based-on Multi-Agent Adaptive Testing System[A]. Xin Li School of Educational Technology Shenyang Normal University Shenyang,Liaoning Province, China Jian Ma School of Educational Technology Shenyang Normal University Shenyang,Liaoning 
Province,China.Proceedings of 2010 Third International Conference on Education Technology and Training(Volume 3)[C]. 2010

[5] Mixed Traffic Information Collection System based on Pressure Sensor[A]. Wenzhe Liao1 1 University of Detroit Mercy,Computer Science Department,Detroit,USA Mingsheng Liu2, and Qingli Meng3 2 School of Information Engineering,Handan Colledge, Handan,China,056005 3 School of Information \& Electrion Engineering,Hebei University of Engineering, Handan,China, 056038.Proceedings of 2010 Second International Asia Symposium on Intelligent Interaction and Affective Computing and 2010 Second International Conference on Innovation Management (ASIA-ICIM 2010)[C]. 2010

[6] "The real-time simulation of cabin automation control system based on iFix OPC communications system". Lingling Yin,Wei Liu,Weiren Shi. Chinese Journal of Scientific Instrument . 2008 [7] S.W.Zhou, W.B.Xie. Design and implementation of smart home terminal based on Android[J]. Microcomputer \& Its Applications, 2012,31(14):10-13.

[7] Application of PLC S7-300 InLarge-Scale Wind Turbine Generator Control System. XinWu, BinZhao, ChunliangE, HonghuaXu. ElectricalAutomation . 2003 